\title{
Fourmis des bois en Suisse: état de la situation et perspectives de monitoring
}

Daniel Cherix

Christian Bernasconi

Arnaud Maeder

Anne Freitag
Département d'écologie et d'évolution de l'Université de Lausanne $(\mathrm{CH})^{*}$

Musée de zoologie de Lausanne (CH)

Musée d'histoire naturelle de La Chaux-de-Fonds (CH)

Musée de zoologie de Lausanne $(\mathrm{CH})$

\section{Wood ants in Switzerland: situation and outlook of a monitoring}

\begin{abstract}
The authors summarise their state of knowledge about wood ants and their role in the forest ecosystem. They also describe the situation in Switzerland, their past development and their conservation status. In several regions, mainly in the Plateau, wood ants seem to diminish, despite their total protection since 1966. The reasons for this regression are not well known, but the fragmentation of forest habitats in the Plateau region and direct damage to ant nests seem to play a certain role. A new project in which the development of wood ant nests is monitored in Swiss forest reserves (Formica-Forêts- $\mathrm{CH}$ ) was recently started in the Swiss national park. It is to be extended, in collaboration with the forest services, over the whole of Switzerland.
\end{abstract}

Keywords: wood ants, formica rufa, forest reserves, monitoring, Switzerland

doi: $10.3188 /$ szf. 2012.0232

* Place de la Riponne 6, CP, CH-1014 Lausanne, courriel daniel.cherix@unil.ch

$\mathrm{L}$ e groupe des fourmis des bois renferme six espèces distinctes en Suisse: Formica rufa Linnaeus, 1758 - Formica polyctena Förster, 1850 Formica pratensis Retzius, 1783 - Formica lugubris Zetterstedt, 1840 - Formica paralugubris Seifert 1996 et Formica aquilonia Yarrow, 1955. Une septième espèce, en cours de description, a été découverte récemment au Parc national suisse (Bernasconi et al 2011). F. rufa et $F$. polyctena sont surtout présentes en plaine en dessous de $800 \mathrm{~m}$ d'altitude (Cherix \& Higashi 1979, Freitag \& Cherix 2009). F. pratensis est également une espèce de plaine, mais elle peut monter à plus de $1000 \mathrm{~m}$ dans les régions bien exposées (Bernasconi non publié). Les trois dernières espèces, $F$. lugubris, $F$. paralugubris et $F$. aquilonia, sont des fourmis montagnardes à alpines. En Suisse, elles s'observent dès $700 \mathrm{~m}$ environ, mais sont surtout abondantes dès 1000 m d'altitude (Cherix \& Higashi 1979, Freitag \& Cherix 2009). F. aquilonia n'a été trouvée avec certitude en Suisse qu'en Engadine (Kutter 1977).

Toutes ces espèces se caractérisent par la construction de fourmilières en forme de dôme plus ou moins élevé constitué de matériel végétal sec (aiguilles de conifères, brindilles, etc.). Elles sont très majoritairement liées au milieu forestier, colonisant les forêts et les lisières (figure 1). F. pratensis fait ex- ception, s'installant surtout dans les prés et les prairies maigres. Avec la disparition progressive de ces milieux naturels, $F$. pratensis se rencontre actuellement surtout sur les talus herbeux en bordure de route (Freitag et al 2008).

Relevons encore deux espèces visibles en Suisse et présentant quelques similarités avec les fourmis des bois: F. truncorum Fabricius, 1804 et $F$. sanguinea Latreille, 1798. La première espèce est largement répandue dans le nord de l'Europe (Rosengren et al 1985, 1986). Elle construit aussi des nids avec du matériel végétal sec. En revanche, ceux-ci sont allongés et très rarement en forme de dôme. Les ouvrières se distinguent des ouvrières du groupe des fourmis des bois par la couleur rouge uniforme de leur tête (bicolore chez les fourmis des bois) et une abondante pilosité sur tout le corps.

F. sanguinea est une espèce bien particulière puisqu'elle fait partie des fourmis esclavagistes (Kutter 1969). Cette espèce se rencontre en milieu forestier et à la lisière des forêts. Ses nids sont souvent construits en partie sur d'anciennes souches avec du matériel végétal sec, mais comme les esclaves (fourmis du genre Formica, sous-genre Serviformica) creusent le sol, il y a aussi de la terre mélangée avec le matériel, ce qui les distingue du nid des fourmis des bois. 
Fig. 1 Fourmilière de fourmis des bois dans une forêt du Parc national suisse.

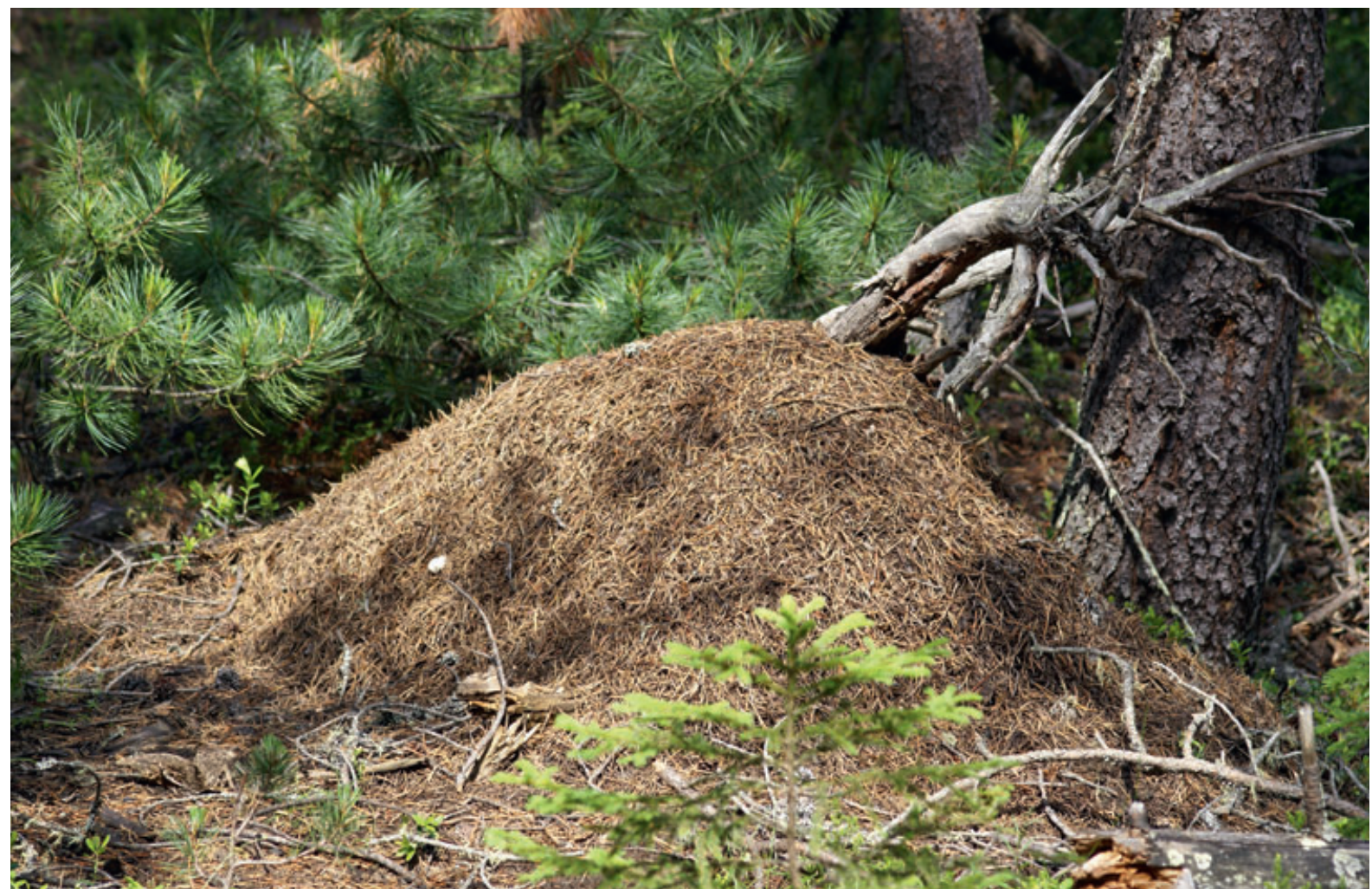

\section{Statut légal}

Les fourmis des bois constituent un groupe particulier de fourmis car elles ont été les premiers insectes protégés en Suisse par la Loi fédérale du

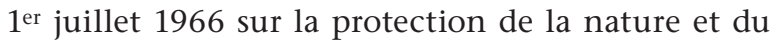
paysage (LPN, RS 451). A l'époque de leur mise sous protection, l'économie forestière était de première importance, et des solutions pour faire face aux problèmes des espèces animales ravageant les forêts étaient recherchées (Gösswald 1951, Pavan 1959). C'est aussi dans ces années-là que différentes études démontrèrent l'effet bénéfique des fourmis des bois sur la diminution des densités de ravageurs forestiers (voir synthèses par Gösswald 1989 et Pavan 1981). La protection des fourmis des bois fut donc décrétée dans le but de protéger les forêts. Plusieurs autres pays, comme l'Allemagne ou l'Autriche, décidèrent aussi de mettre ces insectes sous protection. En Italie, des fourmilières furent même transplantées dans un but de lutte biologique (Carl et al 1976, Pavan 1959, 1976, Ronchetti \& Groppali 1995). De nos jours, une telle pratique mériterait une réflexion scientifique adaptée.

\section{Rôles écologiques}

Les fourmis des bois sont, depuis plus d'un siècle, l'objet de nombreuses études en écologie forestière. Elles ont servi et servent encore de support à de très nombreux travaux en biologie et écologie (voir bibliographies de Cotti 1963, 1996). En raison de leur importance numérique, les fourmis des bois jouent de grands rôles dans les forêts qu'elles occupent (Gösswald 1990, Punttila 1996, Rosengren et al 1979, Savolainen et al 1989). Elles modifient leur habitat en influençant les communautés d'autres fourmis (Savolainen 1990, 1991, Savolainen \& Vepsäläinen 1988, Savolainen et al 1989) et sont de très importants prédateurs d'insectes et autres invertébrés (Cherix \& Bourne 1980, Niemelä et al 1992, Punttila et al 2004, Skinner 1980). D'après des observations menées dans le Jura vaudois, les ouvrières d'une fourmilière de $F$. paralugubris collectent environ 400000 proies pendant une saison d'activité (Cherix 1981). En poids, cela représente environ $1.2 \mathrm{~kg}$ d'invertébrés. Les fourmis des bois sont assez opportunistes et capturent en priorité les espèces les plus fréquentes. Ainsi, elles peuvent contribuer à limiter les populations d'insectes ravageurs (figure 2) - chenilles de papillons ou d'hyménoptères par exemple - lors de pullulations (Karhu 1998).

Elles protègent les pucerons qu'elles exploitent pour récolter le miellat qu'ils produisent à partir de la sève des arbres (Ito \& Higashi 1991, Rosengren \& Sundström 1987; figure 3). Ce comportement rend service à d'autres espèces, comme les abeilles (Wellenstein 1960). L'élevage des pucerons pour le miellat et l'important prélèvement de sève que cela implique ne semblent pas avoir un bilan négatif sur la croissance des arbres. Toutefois, une étude comparative menée dans le Jura vaudois, dans des zones à fortes concentrations de fourmis des bois (10-15 fourmilières/hectare), entre des épicéas exploités par les fourmis des bois pour le miellat de pucerons et des 
dans les forêts de basse altitude. Il y a plus de 40 ans, Kutter (1963) estimait déjà que les colonies de fourmis des bois avaient subi une forte diminution au cours des 50 à 100 dernières années. C'est lui qui initiera un premier monitoring des fourmis des bois dans la région de Stäfa dans le canton de Zurich (Kutter 1970). Entre deux relevés de fourmilières réalisés en 1963 , et 1970 dans une forêt de 100 ha, il note une diminution de $60 \%$ du nombre de fourmilières (26 nids de F. rufa, 17 nids de F. polyctena et 1 de $F$. pratensis en 1963 respectivement 4, 11 et 1 en 1970). Ce même site revisité en 1976 n'abritait plus aucune fourmilière (Cherix 1977). Une autre étude réalisée durant les années 1970 dans le canton de Vaud par Georges Gris (Cherix 1981) montre, dans une zone pourtant protégée (Réserve naturelle du Bois de Chênes, commune de Genolier), une diminution de plus de $20 \%$ des fourmilières entre 1974 et 1978 . Une troisième étude, menée dans le massif forestier du Jorat (région au nord de Lausanne), situé à une altitude moyenne de $800 \mathrm{~m}$, va dans le même sens (Cherix \& Maddalena-Feller 1988, Gleyre 1999). Si 217 fourmilières étaient comptabilisées sur les 1650 ha prospectés entre 1987/89, seules 101 ont été retrouvées dix ans plus tard. Les observations poursuivies ces dernières années dans cette même région indiquent que de nombreuses stations connues en 1998 ont été totalement abandonnées depuis, en partie à cause des dégâts occasionnés par la tempête Lothar en décembre 1999 (Freitag non publié). Une image un peu différente de la situation des fourmis des bois a été mise en évidence par Kissling (1985) dans le canton de Zurich. D'après un sondage effectué auprès des responsables de 92 districts forestiers, 19 personnes considèrent que les effectifs de fourmis des bois ont subi un fort recul, 14 parlent d'un faible recul, 13 n'ont pas observé de changements et 46 estiment que les fourmis des bois sont en augmentation. Si ces données ne sont pas basées sur un recensement effectif, elles reflètent cependant assez bien les tendances que l'on rencontre sur le Plateau suisse. Malgré la disparité géographique et temporelle de ces données à l'échelle de la Suisse, il ressort que dans certaines régions, on assiste à une diminution des effectifs parfois marquée, alors que d'autres régions semblent moins touchées.

Il convient de noter qu'en Suisse les zones d'altitude sont moins touchées que celles de plaine. Ainsi, le suivi de zones à forte densité dans le Jura vaudois (Cherix et al 2006) montre une très grande stabilité dans les populations de fourmis des bois. Il en est de même au Parc national suisse (Cherix et al 2007) où la densité des fourmilières de fourmis des bois est élevée (environ 3 nids/hectare en zones forestières) et où la stabilité des populations est grande (Cherix non publié). Mais comme relevé par Freitag (2008), les méthodes de recensement et les monitorings diffèrent les uns des autres, et il importe de dé- finir une méthodologie commune à tous pour pouvoir dans le futur disposer de données cohérentes.

\section{Listes rouges}

C'est au cours des années 1970 que l'on commença à se soucier de la régression des fourmis des bois, principalement sur le Plateau suisse (Cherix 1977). En Europe durant plusieurs décennies, l'utilisation abusive des fourmis des bois par la récolte des cocons pour l'élevage d'oiseaux (faisans et autres) ou de poissons (Pavan 1981), les pratiques forestières (monocultures, coupes rases, augmentation de l'usage des machines lourdes), l'utilisation massive d'insecticides ou l'urbanisation ont contribué à faire disparaître de nombreuses fourmilières (Gleyre 1999, Kutter 1970). Si les coupes rases et les insecticides n'ont pas été utilisés en Suisse, le recul des populations sur le Plateau est malgré tout important. C'est ainsi que parmi les six espèces de fourmis des bois de Suisse, cinq sont inscrites sur la liste rouge des animaux menacés de l'Union internationale pour la conservation de la nature ${ }^{1}$ et trois - F. rufa, F. polyctena, F. pratensis - sur la liste rouge des espèces menacées en Suisse (Agosti \& Cherix 1994).

\section{Facteurs de régression}

En plaine, les fourmis des bois semblent souffrir du morcellement des forêts: les zones boisées sont souvent de petite taille et sans connexion les unes avec les autres. Les densités en fourmilières sont souvent très faibles (Freitag 2002, 2008), et chaque nid de $F$. rufa ou $F$. polyctena qui disparaît diminue les chances de voir ces espèces se maintenir localement. L'effet exact des pratiques forestières (réduction des travaux forestiers et densification des forêts, ou au contraire intensification locale de l'exploitation) n'est pas connu. Toutefois la progression des ronces dans les milieux forestiers exploités même rationnellement (forêts joratoises, Ville de Lausanne) provoque une très nette diminution des populations de fourmis des bois. En montagne, la situation est moins alarmante (Cherix et al 2007). Toutefois, les travaux forestiers menés sans discernement (machines ou transport des troncs abattus détruisant des nids), la densification des dessertes forestières et l'élargissement ou le bétonnage des chemins (qui occasionnent une perturbation des pistes empruntées par les fourmis entre leurs nids et leurs sites de fourragement) conduisent également à un appauvrissement local en fourmilières. Les causes exactes de la régression des fourmis des bois en Suisse ne sont pas

1 IUCN (2010) IUCN Red List of Threatened Species. Version 2010.4. www.iucnredlist.org (31.3.2011). 
connues et nécessitent encore des études approfondies. Dans cette optique, les réserves forestières représentent un outil de gestion très intéressant pour entreprendre un suivi des populations de fourmis.

Des données sur les facteurs influençant la régression des fourmis des bois existent tout de même. Plusieurs travaux réalisés dans les forêts du nord de l'Europe (Finlande, F. aquilonia) ont permis de montrer que les coupes rases perturbent considérablement les populations de fourmis des bois. Ces interventions entraînent une diminution de la taille des nids, le dérangement provoqué poussant les fourmis à construire de nombreux petits nids dans les alentours des fourmilières d'origine, nids qui sont souvent abandonnés par la suite (Sorvari \& Hakkarainen 2005, Sorvari \& Hakkarainen 2007). Mais surtout, la production des individus reproducteurs est sérieusement affectée par les coupes rases. Ce dernier point est particulièrement important, car il limite les possibilités de dispersion et de production de nouvelles sociétés. Les mêmes auteurs ont aussi montré que les coupes rases avaient une influence sur la taille des ouvrières, résultant de la diminution des ressources (Sorvari \& Hakkarainen 2009). Mais le point le plus négatif observé était la diminution de la température à l'intérieur des fourmilières, trahissant un déséquilibre dans la thermorégulation des nids (voir Cherix et al 2007 pour une revue de la thermorégulation dans les fourmilières de fourmis des bois). Une température constante dans les fourmilières durant la période d'activité est normalement la règle. Une telle diminution entraîne assez rapidement la disparition des fourmilières. A un autre niveau, le travail de Mäki-Petäys et al (2005) montre que la fragmentation du paysage forestier affecte non seulement les paramètres démographiques des populations de fourmis des bois ( $F$. aquilonia et F. lugubris, région de Moscou), mais aussi la structure génétique des populations, soit leur viabilité.

La protection législative semble s'avérer parfois peu efficace, en regard de la régression constante des fourmis des bois (Cherix 1979, Kutter 1970). Pour compenser ce déficit, des efforts ont été entrepris localement pour informer les forestiers ou d'autres groupes non professionnels de l'importance de ces insectes dans les écosystèmes forestiers (Cherix 1977, Cherix et al 2006).

\section{Un nouveau projet au niveau suisse}

La mise sur pied et la création de réserves forestières prend aujourd'hui une importance fondamentale. L'un des points de la stratégie en matière de réserves forestières est la protection particulière d'animaux et de plantes qui sont rares ou menacés au niveau européen ainsi qu'en Suisse. A cela s'ajoute la notion de suivi pour examiner si ces objectifs de conservation sont atteints ou non. Ces deux points tirés des lignes directrices pour une politique suisse en matière de réserves forestières nous permettent de présenter notre projet intitulé Formica-Forêts- $\mathrm{CH}$ (Waldameisen-Waldreservate-CH). Comme signalé plus haut, la protection législative concernant les fourmis des bois semble avoir montré ses limites. Si l'on souhaite avoir une idée plus précise de la situa-
Fig. 4 Relevé des caractéristiques de station autour d'une fourmilière au Parc National Suisse (Projet Formica-Forêts- $\mathrm{CH}$ ).

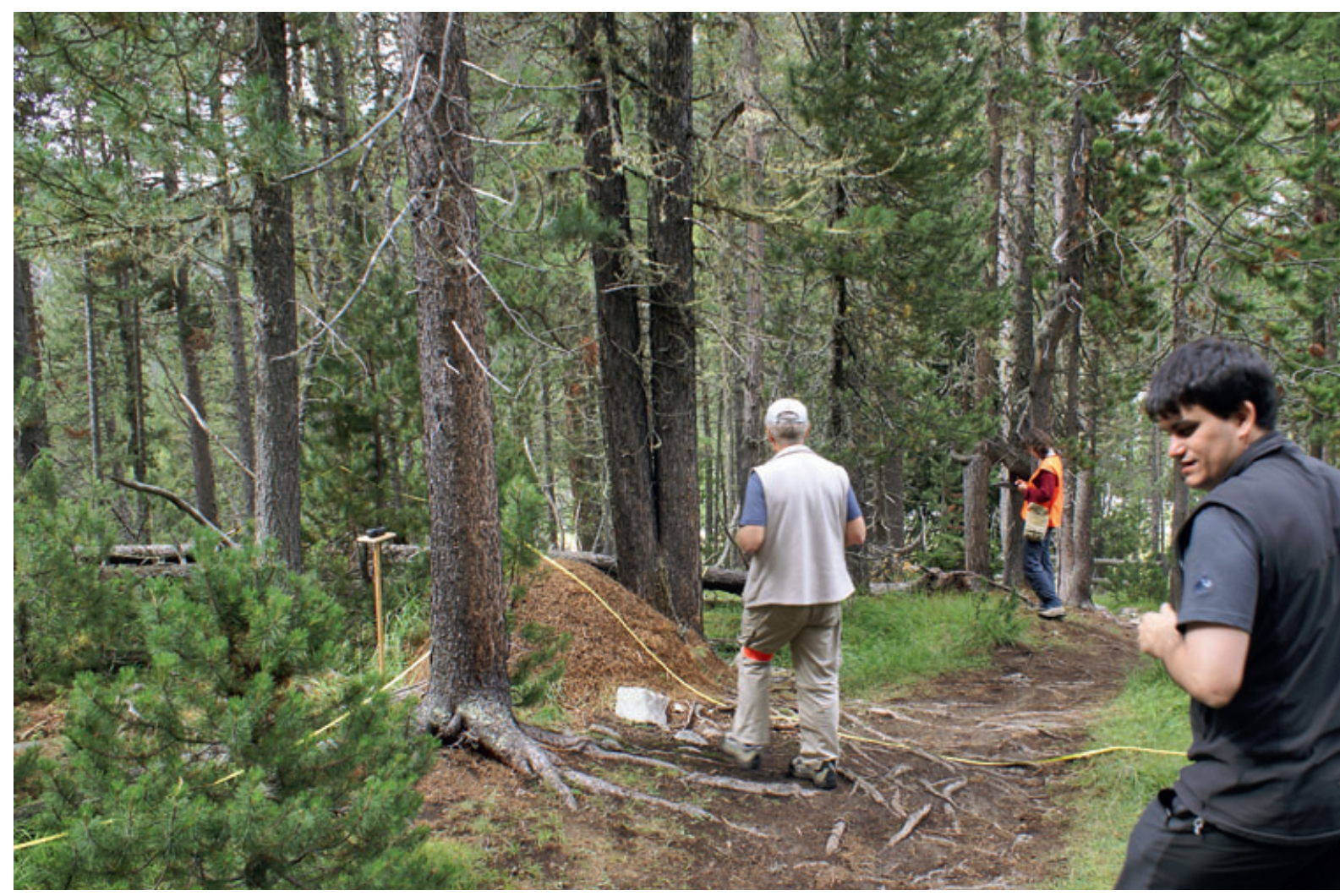


tion et de l'évolution des fourmis des bois en Suisse et proposer des mesures concrètes de protection, seul un monitoring peut apporter des réponses. Ce projet, en cours d'élaboration, va permettre d'analyser les causes principales de la raréfaction, voire de la disparition, des fourmis des bois ainsi que leur maintien ou leur expansion dans les forêts de moyenne et haute altitude. La première phase de test a démarré en 2010 au Parc national suisse (soit la plus grande réserve forestière suisse). Le projet consiste à suivre à l'échelle nationale un certain nombre de fourmilières de fourmis des bois au sein de réserves forestières, de parcs naturels régionaux et de réserves naturelles afin de comprendre l'évolution naturelle des colonies, dans des stations avec ou sans interventions humaines. Dans chaque région d'étude, un échantillon de fourmilières représentant les différentes espèces présentes et illustrant différentes situations environnementales (lisière-pleine forêt, jeune fourmilière-nid mature-nid vieillissant, jeune forêt-vieille forêt, etc.) sera sélectionné. Chaque fourmilière sera suivie individuellement, en répétant les observations d'abord toutes les années, puis à des intervalles de trois, cinq ou dix ans selon la rapidité de modification du milieu (figure 4). A l'échelle de la Suisse, un minimum de 600 stations devra être recensé. La comparaison entre les stations des réserves forestières et celles de zones exploitées tentera d'apporter des réponses sur l'importance réelle de l'exploitation forestière sur les fourmis des bois ainsi que sur les facteurs locaux influençant la durée de vie des fourmilières. Pour cela un certain nombre de paramètres environnementaux sont relevés autour de chaque fourmilière étudiée. Chaque nid est géolocalisé et marqué à l'aide d'un transpondeur enterré. Les nids sont décrits (mesure des dimensions, description de l'emplacement par rapport aux arbres environnants, état de la fourmilière, présence de végétation recouvrante, etc.) et photographiés. L'environnement général des fourmilières est caractérisé dans un rayon de $25 \mathrm{~m}$ (type de milieux, présence de routes ou de chemins, travaux forestiers visibles, perturbations éventuelles). Une description plus détaillée de la nature et de la structure de la forêt dans un rayon de $8 \mathrm{~m}$ autour du nid est réalisée (essences principales présentes, taille et densité des arbres, ouverture de la canopée au-dessus du nid mesurée avec un densiomètre hémisphérique).

Un tel travail, entrepris à l'échelon national, va nécessiter l'aide des services forestiers cantonaux, en particulier pour localiser les fourmilières à échantillonner, voire participer au relevé des données sur le terrain. Il devrait déboucher sur un catalogue de mesures à entreprendre pour assurer la pérennité de ces espèces dont l'importance dans les écosystèmes forestiers est clairement démontrée aujourd'hui. Une collaboration active avec les services forestiers, telle que celle réalisée en Suisse dans les années 1960 (Kut- ter 1970), permettrait d'avancer rapidement et d'obtenir en quelques années des résultats applicables sur le terrain.

Le maintien et le développement des populations de fourmis des bois en Suisse vont dépendre en partie de l'augmentation des surfaces de réserves forestières, mais aussi de la mise en place de nouvelles pratiques forestières, basées sur une analyse actuelle de la situation d'ici quelques années seulement.

Soumis: 4 avril 2011, accepté (avec comité de lecture): 9 mars 2012

\section{Références}

AGOSTI D, CHERIX D (1994) Liste rouge des fourmis menacées de Suisse. In: OFEFP. Listes rouges des espèces animales menacées de Suisse. Berne: Office fédéral de l'environnement forêt paysage. pp. 45-47.

BERNASCONI C, CHERIX D, SEIFERT B, PAMILO P (2011) Molecular taxonomy of the Formica rufa group (red wood ants) (Hymenoptera: Formicidae): a new cryptic species in the Swiss Alps? Myrmecological News 14: 37-47.

CARL KP ET AL (1976) A review of biological control in western and southern Europe. Tech Comm Commonwealth Inst Biol Control 7: 1-182.

CHERIX D (1977) Les fourmis des bois et leur protection. Zurich: WWF Suisse. 32 p.

CHERIX D (1979) Les fourmis rousses (groupe Formica rufa) en Suisse. Rapport sur les connaissances acquises et les données récentes concernant les fourmis rousses en Suisse. Bull OILB/SROP 2: 31-36.

CHERIX D (1981) Contribution à la biologie et à l'écologie de Formica lugubris Zett. (Hymenoptera, Formicidae). Le problème des super-colonies. Lausanne: Univ Lausanne, PhDthesis. 212 p.

CHERIX D, BOURNE JD (1980) A field study on a super-colony of the red wood ant Formica lugubris Zett. in relation to other predatory Arthropodes (Spiders, Harvestmen and ants). Rev Suisse Zool 87: 955-973.

CHERIX D, DEVENOGES A, FREITAG A, BERNASCONI C, MAEDER $A$ (2007) Premier recensement des fourmis des bois (groupe Formica rufa) au Parc national suisse. Nat.park-Forsch Schweiz 94: 69-79.

CHERIX D, FREITAG A, MAEDER A (2006) Les fourmis des bois du Parc jurassien vaudois. St-George: Parc Jurassien Vaudois. 120 p.

CHERIX D, HIGASHI S (1979) Distribution verticale des fourmis dans le Jura vaudois et recensement préliminaire des bourdons (Hymenoptera, Formicidae et Apidae). Bull Soc Vaudoise Sci Nat 356: 316-324.

CHERIX D, MADDALENA-FELLER C (1988) De la fourmi domestique à la fourmi des bois. Petit guide myrmécologique à travers la ville de Lausanne. Lausanne: Service forêts domaines vignobles. $42 \mathrm{p}$.

CHRISTE P, OPPLIGER A, BACANLÀ F, CASTELLA G, CHAPUISAT M (2003) Evidence for collective medication in ants. Ecol Letters 6: 19-22.

COTTI G (1963) Bibliografia ragionata 1930-1961 del gruppo Formica rufa. Collona Verde 8: 1-413.

COTTI G (1996) A bibliography of the Formica rufa-group ( $\mathrm{Hy}$ menoptera, Formicidae). Insect Social Life 1: 133-136.

DE BRUYN GJ, GOOSEN-DE ROO L, HUBREGTSE VAN DEN BERG AIM, FEIJEN HR (1972) Predation of ants by woodpeckers. Ekologia Polska 20: 84-91. 
DEKONINCK W, HENDRICKX F, GROOTAERT P, MAELFAIT FP (2010)

Present conservation status of red wood ants in northwestern Belgium: worse than previously, but not a lost cause. Eur J Entomol 107: 209-218.

DOMISCH TET AL (2008) Decomposition of organic matter and nutrient mineralisation in wood ant (Formica rufa group) mounds in boreal coniferous forests of different age. Biol Fertil Soils 44: 539-545.

FLEISCHMANN E, MURPHY DD, BRUSSARD PF (2000) A new method for selection of umbrella species for conservation planning. Ecol Applic 10: 569-579.

FRANKEL O, SOULE M (1981) Conservation and evolution. Cambridge: Cambridge Univ Press. $327 \mathrm{p}$.

FREITAG A (2002) «FORMIS-2000». Inventaire et protection des fourmis des bois du canton de Vaud. Lausanne: Musée cantonal zoologie. $36 \mathrm{p}$.

FREITAG A (2008) Les fourmis des bois (Hymenoptera, Formicidae) sont-elles en régression en Suisse? Le point sur nos connaissances et réflexions pour des études futures. Entomo Helvetica 1: 33-41.

FREITAG A, CHERIX D (2009) Distribution des fourmis des bois et espèces apparentées (Hymenoptera, Formicidae, genre Formica) dans le canton de Vaud. Entomo Helv 2: 83-95.

FREITAG A, DISCHINGER C, CHERIX D (2008) Formica pratensis (Hyménoptères: Formicidae) dans le canton de Vaud: état des peuplements et importance des talus de routes comme milieu de substitution. Bull Soc vaudoise Sci nat 91: 4768.

GLEYRE M (1999) Bilan du programme Jorlog: état des connaissances faunistiques dans les forêts de la Ville de Lausanne (Jorat). Approche globale de la biodiversité: application d'un SIG. Evolution dans le temps: exemple du groupe Formica rufa. Genève: Univ Genève. 45 p.

GORB SN, GORB EV (1999) Dropping rates of elaiosome-bearing seeds during transport by ants (Formica polyctena Foerst.): Implications for distance dispersal. Acta Oecol 20: 509-518.

GORB SN, GORB EV, PUNTTILA P (2000) Effects of redispersal of seeds by ants on the vegetation pattern in a deciduous forest: A case study. Acta Oecol 21: 293-301.

GösSWALD K (1951) Die rote Waldameise im Dienste der Waldhygiene. Lüneburg: Metta Kinau. 160 p.

GösSWALD K (1989) Die Waldameise. Band 1. Biologische Grundlagen, Ökologie und Verhalten. Wiesbaden: Aula. $660 \mathrm{p}$.

GÖSSWALD K (1990) Die Waldameise. Band 2. Die Waldameise im Ökosystem Wald, ihr Nutzen und ihre Hege. Wiesbaden: Aula. 510 p.

GROSSE C, KACZENSKY P, KNAUER F (2003) Ants: a food source sought by Slovenian brown bears (Ursus arctos)? Can J Zool 81: 1996-2005.

HEINKEN T, ROHNER MS, HOPPERT M (2007) Red wood ants (Formica rufa group) disperse bryophyte and lichen fragments on a local scale. Nova Hedwigia Suppl 131: 147163.

HÖLLDOBLER B, WILSON EO (1990) The ants. Berlin: Springer. $732 \mathrm{p}$.

ITO F, HIGASHI S (1991) An indirect mutualism between oaks and wood ants via aphids. J Animal Ecol 60: 463-470.

KARHU KJ (1998) Effects of ant exclusion during outbreaks of a defoliator and a sap-sucker on birch. Ecol Entomol 23: 185-194.

KILPELÄINEN J ET AL (2007) Carbon, nitrogen and phosphorus dynamics of ant mounds (Formica rufa group) in managed boreal forests of different successional stages. Appl Soil Ecol 36: 156-163.
KISSLING E (1985) Untersuchungen über die Biotopansprüche und einen allfälligen Rückgang von Roten Waldameisen aus der Formica rufa-Gruppe in der Schweiz. Zürich: ETH Zürich, PhD-thesis. 76 p.

KUTTER H (1963) Ein kleiner Beitrag zur Kenntnis unserer Waldameisenfauna. Schweiz Z Forstwes 114: 646-653.

KUTTER H (1969) Die sozialparasitischen Ameisen der Schweiz. Neujahrsblatt. Naturforsch Ges Zürich 171: 5-62.

KUTTER H (1970) Ein kleiner Beitrag zum Naturschutzjahr 1970. Schweiz Z Forstwes 121: 835-838.

KUTTER H (1977) Formicidae-Hymenoptera. Zürich: Insecta Helvetica, 2. ed. $298 \mathrm{p}$.

LAAKSO J, SETÄLÄ H (1997) Nest mounds of red wood ants (Formica aquilonia): hot spots for litter-dwelling earthworms. Oecologia 111: 565-569.

LAAKSO J, SETÄLÄ H (1998) Composition and trophic structure of detrital food web in ant nest mounds of Formica aquilonia and in the surrounding forest soil. Oikos 81: 266-278.

LENOIR L, BENGTSSON J, PERSSON T (1999) Effects of coniferous resin on fungal biomass and mineralisation processes in wood ant nest materials. Biol Fertil Soils 30: 251-257.

LENOIR L, PERSSON T, BENGTSSON J (2001) Wood ant nests as potential hot spots for carbon and nitrogen mineralisation. Biol Fertil Soils 34: 235-240.

MÄKI-PETÄYS H, ZAKHAROV A, VILJAKAINEN L, CORANDER J, PAMILO P (2005) Genetic changes associated to declining populations of Formica ants in fragmented forest landscape. Molec Ecol 14: 733-742.

NIEMELÄ P, HAILA H, HALME E, PAJUNEN T, PUNTTILA P (1992) Small-scale heterogeneity in the spatial distribution of carabid beetles in the southern Finnish taiga. J Biogeogr 19: 173-181.

PAVAN M (1959) Attivitá italiana per la lotta biologica con formiche del gruppo Formica rufa contro gli inetti dannosi alle foreste. Collona Verde 4: 1-79.

PAVAN M (1976) Utilizzazione delle formiche del gruppo Formica rufa nella difesa biologica delle foreste. Collona Verde 39: 175-197.

PAVAN M (1981) Utilizzazione delle formiche del gruppo Formica rufa nella difesa biologica delle foreste. Collana verde 59: 185-223.

POKARZHEVSKIJ AD (1981) The distribution and accumulation of nutrients in nests of ant Formica polyctena (Hymenoptera: Formicidae). Pedobiologia 21: 118-124.

PUNTTILA P (1996) Succession, forest fragmentation, and the distribution of wood ants. Oikos 75: 291-298.

PUNTTILA P, NIEMELÄ P, KARHU K (2004) The impact of wood ants (Hymenoptera: Formicidae) on the structure of invertebrate community on mountain birch (Betula pubescens ssp. czerepanovii). Ann Zool Fenn 41: 429-446.

ROBERGE JM (2006) Umbrella species as a conservation planning tool. An assessment using resident birds in hemiboreal and boreal forests. Uppsala: Swed Univ Agric Sci, PhDthesis. $34 \mathrm{p}$.

RONCHETTI G, GROPPALI R (1995) Quarantacinque anni di protezione forestale con Formica lugubris Zett. (Hym. Formicidae). L'esperienza di monte d'Alpe (Appennino Ligure in Provincia di Pavia). Roma: Ministero Università ricerca scientifica tecnologica. $256 \mathrm{p}$.

ROSENGREN R, VEPSÄLÄINEN K, WUORENRINNE H (1979) Distribution, nest densities, and ecological significance of wood ants (the Formica rufa group) in Finland. Bull OILB/ SROP II-3: 183-213.

ROSENGREN R, CHERIX D, PAMILO P (1985) Insular ecology of the red wood ant Formica truncorum Fabr. I. Polydomous nesting, population size and foraging. Mitt Schweiz entomol Ges 58: 147-175. 
ROSENGREN R, CHERIX D, PAMILO P (1986) Insular ecology of the red wood ant Formica truncorum Fabr. II. Distribution, reproductive strategy and competition. Mitt Schweiz entomol Ges 59: 63-94.

ROSENGREN R, SUNDSTRÖM L (1987) The foraging system of a red wood ant colony (Formica s.str.). Collecting and defending food through an extended phenotype. Exper suppl 54: 117-137.

SAVOLAINEN R (1990) Colony success of the submissive ant Formica fusca within territories of the dominant Formica polyctena. Ecol Entomol 15: 79-85.

SAVOLAINEN R (1991) Interference by wood ant influences size selection and retrieval rate of prey by Formica fusca. Behav Ecol Sociobiol 28: 1-7.

SAVOLAINEN R, VEPSÄLÄINEN K (1988) A competition hierarchy among boreal ants: impact on resource partitioning and community structure. Oikos 51: 135-155.

SAVOLAINEN R, VEPSÄLÄINEN K, WUORENRINNE H (1989) Ant assemblages in the taiga biome: testing the role of territorial wood ants. Oecologia 81: 481-486.
SKINNER GJ (1980) The feeding habits of the wood ant Formica rufa (Hymenoptera: Formicidae) in limestone woodland in North-west England. J Animal Ecol 49: 417-433.

SORVARI J, HAKKARAINEN H (2005) Deforestation reduces nest mound size and decreases the production of sexual offspring in the wood ant Formica aquilonia. Ann Zool Fenn 42: 259-267.

SORVARI J, HAKKARAINEN H (2007) Wood ants are wood ants: deforestation causes population declines in the polydomous wood ant Formica aquilonia. Ecol Entomol 32: 707711.

SORVARI J, HAKKARAINEN H (2009) Forest clear-cutting causes small workers in the polydomous wood ant Formica aquilonia. Ann Zool Fenn 46: 431-438.

SWENSON JE, JANSSON A, RIIG R, SANDEGREN F (1999) Bears and ants: myrmecophagy by brown bears in central Scandinavia. Can J Zool 77: 551-561.

WELLENSTEIN G (1960) Ergebnisse vierjähriger Untersuchungen über die Steigerung der Waldbienentracht. Z Angew Entomol 47: 32-41.

\section{Fourmis des bois en Suisse: état de la situation et perspectives de monitoring}

Les auteurs présentent un résumé des connaissances actuelles sur les fourmis des bois et leur importance dans les écosystèmes forestiers. Ils abordent aussi leur situation en Suisse du point de vue de leur évolution récente et leur protection. Dans plusieurs régions, surtout en plaine, les fourmis des bois semblent être en régression, malgré leur protection intégrale depuis 1966. Les causes de cette diminution sont mal connues, mais le morcellement de l'habitat forestier sur le Plateau suisse et des dégâts directs causés aux fourmilières jouent un certain rôle. Un projet de monitoring et de suivi de l'évolution de fourmilières de fourmis des bois en Suisse dans les réserves forestières (Formica-Forêts- $\mathrm{CH}$ ) vient de démarrer au Parc national suisse et devrait s'étendre à l'échelle de la Suisse en collaboration avec les services forestiers.

\section{Waldameisen in der Schweiz: aktuelle Situation und Ausblick hinsichtlich eines Monitorings}

Die Autoren fassen den Kenntnisstand zu den Waldameisen und zu ihrer Bedeutung in Waldökosystemen zusammen. Sie stellen auch deren Situation in der Schweiz, deren vergangene Entwicklung sowie den Schutzstatus dar. In mehreren Regionen vor allem des Mittellandes scheint die Zahl der Waldameisen zurückzugehen, obwohl sie seit 1966 integral geschützt sind. Die Ursachen dieses Rückganges sind wenig bekannt, aber die Zerstückelung der Waldhabitate im Mittelland und direkte Schäden an den Ameisenhaufen spielen eine gewisse Rolle. Ein neues Monitoringprojekt zur Entwicklung der Waldameisenhaufen in Schweizer Waldreservaten (Waldameisen-Waldreservate- $\mathrm{CH}$ ) wurde kürzlich im Schweizerischen Nationalpark gestartet. Es soll in Zusammenarbeit mit den Forstdiensten auf die ganze Schweiz ausgedehnt werden. 